\title{
Tendências da aquisição lexical em crianças em desenvolvimento normal e crianças com Alterações Específicas no Desenvolvimento da Linguagem
}

\author{
Trends on lexical acquisition in children within normal \\ development and children with developmental language \\ disorder
}

\author{
Juliana Perina Gândara ${ }^{1}$, Debora Maria Befi-Lopes ${ }^{2}$
}

\begin{abstract}
RESUMO
O objetivo do presente estudo foi descrever as semelhanças e diferenças encontradas ao longo da aquisição lexical por crianças em desenvolvimento normal e crianças com Alterações Específicas no Desenvolvimento da Linguagem (AEDL), por meio de um amplo levantamento bibliográfico em bases de dados (SciELO, Lilacs, PubMed, Web of Science, Dedalus), que abrangeu as últimas décadas de estudos na área. Os estudos selecionados, de natureza observacional ou experimental, mostraram grande variedade de achados relacionados ao desenvolvimento do vocabulário, abrangendo as tendências e variações e também outras habilidades envolvidas no processo de aquisição lexical. De maneira geral, os resultados sugerem que as alterações lexicais que constituem um dos marcos inicialmente observados em crianças com AEDL são justificadas por dificuldades observadas em habilidades e/ou características influenciadas ou diretamente relacionadas aos mecanismos envolvidos no processamento da informação, que comprometem a qualidade e a recuperação das representações fonológicas e semânticas correspondentes a um novo item lexical. Entretanto, vários estudos sugerem que situações ostensivas e de grande suporte contextual que enfoquem poucas novas palavras favorecem a aquisição lexical de crianças com AEDL.
\end{abstract}

Descritores: Linguagem infantil; Desenvolvimento da linguagem; Transtornos do desenvolvimento da linguagem; Vocabulário; Literatura de revisão como assunto

\section{INTRODUÇÃO}

Tradicionalmente, a descrição da aquisição da linguagem é feita com base em alguns marcos observáveis do desenvolvimento gramatical de crianças em desenvolvimento normal. A partir desses marcos, os diversos aspectos da linguagem (fonologia, semântica, morfossintaxe, pragmática) podem ser estudados tanto separadamente quanto nas suas relações, já

Trabalho realizado no Laboratório de Investigação Fonoaudiológica em Desenvolvimento da Linguagem e suas Alterações do Departamento de Fisioterapia, Fonoaudiologia e Terapia Ocupacional da Faculdade de Medicina da Universidade de São Paulo - USP - São Paulo (SP), Brasil.

(1) Doutora, Colaboradora do Laboratório de Investigação Fonoaudiológica em Desenvolvimento da Linguagem e suas Alterações do Departamento de Fisioterapia, Fonoaudiologia e Terapia Ocupacional da Faculdade de Medicina da Universidade de São Paulo - USP - São Paulo (SP), Brasil.

(2) Livre-docente, Professora associada do Curso de Fonoaudiologia do Departamento de Fisioterapia, Fonoaudiologia e Terapia Ocupacional da Faculdade de Medicina da Universidade de São Paulo - USP - São Paulo (SP), Brasil. Endereço para correspondência: Juliana Perina Gândara. R. Cipotânea, 51, Cidade Universitária, São Paulo (SP), Brasil, CEP: 05360-160. E-mail: jugandara@gmail.com

Recebido em: 20/3/2010; Aceito em: 30/4/2010 que todos esses aspectos estão intimamente ligados ao longo do desenvolvimento linguístico ${ }^{(1)}$. A aquisição de palavras constitui um passo fundamental para o posterior desenvolvimento sintático, além de marcar o início da possibilidade de comunicação oral efetiva entre a criança em desenvolvimento e o mundo que a cerca.

A aquisição do vocabulário também apresenta marcos característicos: durante o desenvolvimento normal de linguagem, as primeiras palavras são adquiridas por volta dos 12 meses (entre 10 e 13 meses), seguindo-se um período de desenvolvimento lento e gradual do vocabulário produtivo, num ritmo de aproximadamente 10 palavras por mês até os 18 meses. Conforme a criança se aproxima do marco da produção de 50 palavras, a velocidade de crescimento aumenta (caracterizando a chamada "explosão" do vocabulário), sendo que muitas palavras podem ser adquiridas por dia após um número mínimo de exposições. Na idade escolar, o desenvolvimento lexical apresenta picos de desenvolvimento acelerado, característica que permanece até por volta dos 16 anos. Na fase adulta, o léxico continua a aumentar, mas o desenvolvimento passa a ser mais lento e dependente de fatores sociais e ocupacionais ${ }^{(2)}$. 
Em crianças com Alterações Específicas no Desenvolvimento da Linguagem (AEDL), cujo diagnóstico é realizado por meio de critérios de exclusão - as dificuldades são específicas da linguagem, eliminando possíveis fatores causais, como lesões neurológicas marcadas, perdas auditivas, rebaixamento intelectual, privação neurossensorial e comprometimentos emocionais de ordem abrangente ${ }^{(3)}$-, tais características de aquisição lexical podem estar alteradas.

As AEDL, embora apresentem grande heterogeneidade entre os sujeitos e na evolução de um mesmo sujeito, são inicialmente caracterizadas por atrasos na aquisição da linguagem e déficits semânticos significativos, observados por um vocabulário restrito $^{(4)}$. Em geral, elas apresentam atraso no desenvolvimento das primeiras palavras e falha na expansão vocabular (que ocorre, no desenvolvimento normal, entre os 18 e os 24 meses), dificuldades em adquirir conceitos abstratos e em combinar os significados das palavras para formar sentenças. É comum também que apresentem alterações fonológicas importantes, manifestadas por um discurso ininteligível, e a partir dos três anos mostram alterações morfológicas e sintáticas, com uso de sentenças de menor complexidade, uso limitado de subordinação, dificuldades no uso dos marcadores de tempo e número verbais, e omissão ou uso inadequado de elementos gramaticais obrigatórios, como artigos, pronomes e plural dos morfemas ${ }^{(5)}$.

Assim, as dificuldades lexicais iniciais, que se manifestam na aquisição de substantivos e verbos de ação, posteriormente se refletem nas alterações morfossintáticas, que se constituem uma das principais marcas linguísticas do Distúrbio Específico de Linguagem $(\mathrm{DEL})^{(6)}$. As dificuldades na elaboração linguística geralmente levam a manifestações secundárias no uso da linguagem, apresentando menor intenção comunicativa e maior uso do meio comunicativo não-verbal ${ }^{(7)}$.

Nas últimas décadas, muitos estudos têm enfocado a aquisição lexical e as habilidades semânticas de crianças com Alterações Específicas no Desenvolvimento da Linguagem (AEDL). Embora essas pesquisas tenham abordagens e enfoques diferentes, com base em estudos sobre o desenvolvimento normal do vocabulário, as evidências têm mostrado que essas crianças de fato apresentam desenvolvimento mais lento com relação à emergência das primeiras palavras e aos progressos subsequentes, além de cometerem mais erros lexicais do que as crianças em desenvolvimento normal de linguagem ${ }^{(4)}$.

O objetivo do presente estudo foi descrever as semelhanças e diferenças encontradas ao longo da aquisição lexical por crianças em desenvolvimento normal e crianças com AEDL. Para isso, foi realizado um amplo levantamento bibliográfico em bases de dados (SciELO, Lilacs, PubMed, Web of Science, Dedalus), que abrangeu um as últimas décadas de estudos na área; os estudos foram localizados e compilados sinteticamente.

\section{REVISÃO DA LITERATURA}

\section{Variações individuais e tendências na aquisição lexical}

Estudos observacionais e experimentais mais atuais sobre a aquisição lexical têm enfocado o rápido crescimento do vocabulário no início do desenvolvimento da linguagem e as características que o influenciam, além das semelhanças e diferenças individuais ao longo do processo de aquisição, tanto em crianças com desenvolvimento normal de linguagem quanto em crianças com alterações de linguagem, de qualquer natureza. Tais estudos têm como objetivo não só descrever e compreender as variações individuais no desenvolvimento normal de linguagem, mas também verificar o que ocorre quando as crianças ultrapassam o que pode ser considerado variação individual, caracterizando alterações no desenvolvimento do vocabulário e da linguagem de maneira geral.

Um estudo com crianças em desenvolvimento normal de linguagem ${ }^{(8)}$ observou a existência de variações individuais nos primeiros estágios do desenvolvimento lexical, especificamente em relação ao número de palavras adquiridas em cada idade. Assim, as crianças apresentam uma fase inicial de aquisição lexical lenta e, em seguida, uma fase rápida, durante a qual muitas palavras são incorporadas por dia; entretanto, segundo esses autores, há certa variação individual em relação às idades em que tais marcos ocorrem.

Uma das tendências da aquisição lexical está relacionada ao referencial das palavras aprendidas: as crianças têm maior facilidade em adquirir substantivos, verbos e adjetivos, palavras de classe aberta que apresentam, em geral, referenciais mais concretos e por isso são mais facilmente aprendidas pelo contexto ${ }^{(9,10)}$. Alguns estudos mostraram que o número de palavras referentes a objetos é maior do que o número de palavras relativas a ações ${ }^{(2)}$; entretanto, Schwartz e Leonard ${ }^{(11)}$ ressaltaram que essa tendência é observada quando o repertório das crianças tem entre 10 e 75 palavras, mesmo quando a exposição aos dois tipos de palavras (objetos e ações) é controlada.

A aquisição de nomes de objetos parece ser o método mais comum pelo qual as crianças adicionam um grande número de palavras a seu vocabulário num período relativamente curto de tempo ${ }^{(4)}$; entretanto, existem algumas controvérsias com relação à natureza universal dessa tendência ${ }^{(10)}$.

Outra característica da aquisição lexical refere-se à influência da frequência com que uma palavra é utilizada na língua. Pesquisas indicam que palavras de alta frequência tendem a ser reconhecidas mais rapidamente e identificadas com maior precisão do que as palavras de baixa frequência. Da mesma forma, as palavras de alta frequência são produzidas mais rapidamente e são menos sujeitas a $\operatorname{erros}^{(12)}$.

No início do desenvolvimento de linguagem, a aquisição lexical também sofre restrições baseadas na fonologia das palavras: as crianças mostram maior facilidade em compreender e produzir palavras que começam com fonemas já presentes anteriormente em seu inventário fonológico ${ }^{(1,13)}$. Os padrões de seleção e evitação observados parecem basear-se também na estrutura silábica das palavras produzidas pelos adultos (Ingram, 1974*) ${ }^{(13)}$.

As características fonológicas de seleção lexical transformam-se, com o tempo, de três maneiras: o comprimento, em sílabas, das palavras aumenta; os itens lexicais vão pro-

\footnotetext{
* Ingram D. Phonological rules in young children. J Child Lang. 1974;1:49-64.
} 
gressivamente incluindo mais encontros consonantais; e as preferências por palavras que contêm fonemas específicos, particulares para cada criança, mudam ${ }^{(14)}$.

Um estudo ${ }^{(14)}$ investigou a persistência dos critérios de seleção fonológica na aquisição lexical de crianças pequenas (entre dois e cinco anos), e concluíram que tais critérios, descritos por vários autores, não permanecem muito além do início das produções de mais de uma palavra. Os resultados mostraram-se consistentes com a hipótese de outro estudo ${ }^{(13)}$, de que os critérios fonológicos de seleção lexical operam nas fases primárias do desenvolvimento da linguagem. Além disso, os autores ${ }^{(14)}$ sugerem que outros fatores, além do fonológico, podem influenciar as escolhas de palavras, tais como aspectos sintáticos, pragmáticos e lexicais e propriedades específicas da língua falada pela criança.

Outra tendência largamente documentada ${ }^{(2,15-18)}$ refere-se à precedência da compreensão de novas palavras em relação à produção das mesmas. Diferenças de idade no desenvolvimento dessas duas habilidades foram observadas ${ }^{(2)}$ : as crianças mostraram um intervalo de aproximadamente cinco meses entre a compreensão e a produção de 50 palavras. Esses dados confirmam hipóteses anteriores de que a compreensão se desenvolve antes da produção e de maneira mais rápida ${ }^{(15)}$, e estão de acordo com os resultados de um conjunto de experimentos mais recentes ${ }^{(19)}$, que mostraram que as habilidades das crianças em classificar objetos numa ordem espacial ou temporal são independentes de avanços no crescimento do vocabulário produtivo. Isso sugere que, embora o aprendizado de categorias de objetos possa ser a base do desenvolvimento observado na categorização e nomeação, estas habilidades dependem também de outros fatores, como as experiências pessoais da criança e o contexto particular no qual determinado comportamento é exibido.

Assim, a aquisição de palavras é resultado da interação de diferentes capacidades humanas: sociais, conceituais e linguísticas. Bloom ${ }^{(9)}$ resumiu os princípios que regem a aquisição dos significados das palavras da seguinte maneira: as crianças dividem as sentenças que ouvem em palavras distintas e são capazes de compreender as intenções de seus interlocutores para identificar ao que se referem quando usam as palavras; elas entendem o mundo como um conjunto de propriedades, eventos e processos, e, principalmente, objetos; elas são capazes de generalizar as palavras que aprendem para outros objetos e ações e conseguem distinguir as palavras que se referem a indivíduos e não a categorias (como pronomes e nomes próprios). Tais habilidades melhoram com o desenvolvimento, e as crianças passam a utilizar também o contexto linguístico, explorando propriedades sintáticas e semânticas para adquirir novas palavras.

\section{Influência de fatores lexicais e semânticos na aquisição de novas palavras}

Embora o crescimento do vocabulário, inicialmente, seja bastante lento, com a incorporação de uma ou duas novas palavras por semana até o marco de 50 palavras, por volta dos 18 meses ocorre a "explosão" do vocabulário, e as crianças passam a produzir até nove palavras novas por $\operatorname{dia}^{(2,9)}$. Aos dois anos de idade as crianças são capazes de produzir mais de 200 palavras, e aos dois anos e seis meses, mais de 500 palavras ${ }^{(8)}$.

Quando uma criança se depara com uma palavra desconhecida, vários tipos de informação estão disponíveis para que ela a adquira: informações sobre o seu referente, suas características fonéticas, a estrutura sintática na qual a nova palavra está inserida, as características contextuais da sua ocorrência, e suas relações com palavras adquiridas anteriormente em qualquer uma dessas dimensões ${ }^{(16)}$. Diante desse fato, os pesquisadores têm investido em propor e testar hipóteses que justifiquem e expliquem o grande e rápido aumento do vocabulário.

Alguns autores ${ }^{(20)}$ atribuem a mudança da fase lenta para a rápida de aquisição lexical à mudança da aquisição de "proto-palavras" (referentes ao pareamento de um padrão sonoro inespecífico e um objeto específico, após apresentações simultâneas repetitivas) para a aquisição das palavras "genuínas" (referentes ao pareamento imediato de uma sequência fonética específica e uma categoria de objetos não nomeados). Assim, a "explosão" do vocabulário assinalaria a mudança de um modo de aquisição simplesmente associativo para um modo referencial, no qual a linguagem seria uma forma de representar o mundo.

Outros autores ${ }^{(21)}$ sugerem que a passagem da fase lenta para a fase rápida de aquisição lexical demonstra a compreensão de que todas as coisas podem ser categorizadas. Tal categorização reflete o conhecimento de mundo e é caracterizada por uma estrutura hierárquica, que parte do nível básico (em que há um equilíbrio entre os extremos semelhança e distinção) e "sobe" ou "desce" na hierarquia conforme a especificidade dos dois extremos diminuem (nível mais geral) ou aumentam (nível mais específico) ${ }^{(22)}$.

Já aos três anos as crianças são capazes de classificar os mesmo objetos em diferentes categorias, demonstrando a mesma flexibilidade que adultos no uso dessas categorias para inferências indutivas ${ }^{(22)}$. Essa habilidade é aprendida a partir dos contextos sociais e pragmáticos nos quais as novas palavras são introduzidas para determinar seus significados ${ }^{(23)}$. De acordo com outro estudo ${ }^{(21)}$, a aquisição dessa habilidade cognitiva está relacionada à exposição ao input linguístico (em termos de quantidade e natureza) e facilita o desenvolvimento subsequente de outras habilidades linguísticas, como a aquisição lexical.

As crianças usam as características semânticas de palavras que já conhecem para adicionar novos itens lexicais. Assim, elas criam redes de traços semânticos que variam em quantidade de características conectadas: as redes com maior número de traços semânticos representados facilitam a incorporação de novos itens lexicais, quando comparadas às redes com menos representações ${ }^{(24)}$.

Para que uma criança aprenda uma palavra, os traços semânticos devem ser codificados e associados a uma forma fonológica. Dessa forma, as palavras também podem ser armazenadas e organizadas de acordo com suas características fonológicas. A rede de relações criadas entre as palavras com base nas semelhanças fonológicas é referida na literatura como "vizinhança lexical". A vizinhança lexical das palavras pode facilitar ou dificultar a aquisição de novas palavras dependendo da sua densidade. A densidade da vizinhança lexical é determi- 
nada pela quantidade de palavras reais que podem ser criadas quando um fonema é adicionado, deletado ou modificado. Assim, uma vizinhança lexical é densa quando apresenta muitas palavras que se diferenciam entre si por apenas um fonema, e esparsa quando existem poucas palavras armazenadas com fonologia semelhante ${ }^{(12,18,25,26)}$.

Um estudo ${ }^{(27)}$ sugere que, conforme aumenta a necessidade de incorporar novas palavras ao léxico, as crianças são forçadas a adicionar outras informações fonológicas para criar novas representações lexicais e diferenciar palavras com fonologia semelhante. Assim, o crescimento do vocabulário é responsável pela crescente especificação das representações fonológicas ${ }^{(28)}$.

Em pesquisas sobre acesso lexical e reconhecimento de palavras, os resultados indicam que a competição entre as palavras de vizinhança densa dificulta as respostas nesses tipos de tarefas. Dessa forma, no início da aquisição, as crianças parecem apresentar maior facilidade para reconhecer, acessar e repetir palavras de vizinhança esparsa ${ }^{(12,25)}$. As palavras de vizinhança mais densa são melhor acessadas, reconhecidas e imitadas com a idade e o aumento do léxico ${ }^{(25)}$.

\section{O paradigma do mapeamento rápido}

Qualquer que seja a população estudada, os estudos experimentais sobre aquisição lexical são desenvolvidos de forma a reproduzir ou manipular ou ainda testar variáveis e/ou os princípios operacionais que influenciam o processamento da linguagem e a aprendizagem de novas palavras ${ }^{(29)}$.

Os estudos fundamentais, que basearam vários outros estudos experimentais em aquisição lexical ${ }^{(16,18,29,30)}$, foram desenvolvidos por Carey** e Carey e Bartlett***. Esses autores estudaram a aquisição lexical de crianças em idade pré-escolar e verificaram que, aos três anos, elas pareciam aprender os significados das novas palavras contrastando-as com outras palavras já conhecidas, mesmo após uma ou muito poucas exposições. Carey**** propôs a existência de duas fases de aprendizagem de palavras, uma fase rápida inicial (mapeamento rápido) e uma fase lenta posterior (mapeamento lento), que se diferenciariam de acordo com a quantidade e a qualidade das representações associadas à nova palavra. Assim, o mapeamento rápido seria o conhecimento parcial, o link inicial entre a palavra e o referente, que ocorre quando a criança nota um novo item lexical e rapidamente apreende algum tipo de informação sobre ele após poucas experiências. Por sua vez, o mapeamento lento, fase mais extensa da aquisição lexical, seria o refinamento gradual da representação inicialmente mapeada, com a adição de novas informações, conforme a criança tem outras experiências com a nova palavra ${ }^{(16,18)}$.

A partir dos estudos de Carey e Bartlett, dois tipos básicos de pesquisas experimentais têm sido desenvolvidos: um que investiga os princípios que levam as crianças a interpretar as palavras de uma determinada maneira (em geral, procedimentos experimentais não-ostensivos e em contextos ambíguos) e outro que enfoca como os processos gerais de aprendizagem e memória subsidiam a aquisição lexical (procedimentos experimentais ostensivos em contextos não-ambíguos) ${ }^{(18)}$.

De acordo com o princípio N3C (Novel Name-Nameless Category), testado em um estudo ${ }^{(29)}$ por meio de uma tarefa de mapeamento rápido e outra de categorização, já por volta dos 18 meses, quando o vocabulário infantil passa a crescer rapidamente, as crianças são capazes de inferir sobre uma palavra desconhecida, quando expostas ao seu referente igualmente desconhecido e um outro referente conhecido, ou seja, elas são capazes de mapear o nome desconhecido ao seu referente com base no seu conhecimento anterior da categoria a que $\mathrm{o}$ objeto conhecido pertence.

Esse princípio está relacionado ao princípio da exclusividade mútua ${ }^{(31)}$, segundo o qual a criança assume que a cada objeto corresponde um nome e, portanto, numa tarefa em que são apresentados um objeto familiar e outro não-familiar, o nome desconhecido refere-se ao segundo. Os resultados de um estudo de 2004 sugeriram que esse princípio só se aplica quando novos referentes, não categorizados, são apresentados ${ }^{(26)}$.

Um estudo mais recente ${ }^{(32)}$ estudou a sensibilidade de crianças de 18 meses à ocorrência estatística de palavras desconhecidas se relacionarem a um objeto desconhecido, comparando situações em que o referente era associado à palavra correta em 100\% das apresentações, ou que o referente era associado à palavra correta em $80 \%$ das apresentações e a outra palavra nas $20 \%$ restantes. Os resultados mostraram que os sujeitos foram capazes de usar as informações correlacionadas de maneira imperfeita com seus rótulos para adquirir os significados das novas palavras. Além disso, as crianças demonstraram codificar múltiplos mapeamentos, estocando também informações sobre as palavras de baixa ocorrência durante a apresentação dos referentes, o que permitiria a aplicação do princípio da exclusividade mútua de maneira gradual. Assim, as hipóteses que as crianças criam sobre o possível significado de uma palavra podem ser restritas por esse princípio, assim como pelo contexto sintático e pela compreensão da intencionalidade do interlocutor.

Bloom ${ }^{(9)}$ enfatizou que a habilidade de mapeamento rápido envolve a apreensão tanto de informações semânticas/conceptuais como de traços fonológicos das novas palavras, sendo que as representações semânticas criadas dependem das direções pragmáticas fornecidas pelos interlocutores. Essa sugestão foi corroborada por outro estudo ${ }^{(17)}$, que observou que o uso das pistas contextuais, em especial daquelas referentes às formas dos objetos, facilitou a recuperação das formas fonológicas das novas palavras, sugerindo que essa habilidade é positivamente influenciada pela representação semântica.

Outros autores ${ }^{(33)}$ exploraram a habilidade das crianças de nomear espontaneamente objetos desconhecidos como uma maneira de estudar o acesso lexical. Eles observaram que as crianças frequentemente utilizavam informações sobre a forma dos novos objetos para sua categorização, mesmo sem o su-

\footnotetext{
** Carey S. The child as a word learner. In: Halle M, Bresnan J, Miller GA, editors. Linguistic theory and psychological reality. Cambridge, MA: MIT Press; 1978. cap. 8, p. 264-93.

*** Carey S, Bartlett E. Acquiring a single new word. Papers and Reports on Child Language Development.1978;15:17-29.

***** Carey S. Conceptual change in childhood. Cambridge, MA: MIT Press; 1985.
} 
porte explícito de uma tarefa de nomeação. Em outro estudo, embora a forma dos objetos fosse utilizada como informação para a categorização de objetos (não para a nomeação), esse recurso era ou não considerado pelos sujeitos dependendo da intencionalidade inferida por eles para a criação desses objetos $^{(34)}$.

O conhecimento das relações semânticas entre as palavras também é um aspecto crítico para a apreensão de significa$\operatorname{dos}^{(30)}$. Essas relações incluem inferências lógicas sobre as categorias das palavras, como relações de inclusão, sobreposição e exclusão. A aprendizagem dessas relações foram estudadas em um experimento com crianças de quatro a sete anos de idade ${ }^{(30)}$. Os resultados mostraram que as crianças mais velhas, de seis e sete anos, aprenderam mais palavras e relações semânticas corretas do que as crianças mais novas (quatro e cinco anos). Esse dado sugere que, embora os préescolares apresentem grande habilidade para adquirir novas palavras, as relações semânticas estabelecidas entre elas são fracas, corroborando os estudos sobre as fases rápida e lenta de mapeamento de novas palavras ${ }^{(16,18)}$. Além disso, a relação de exclusão entre as palavras foi mais facilmente aprendida do que as relações de inclusão e sobreposição, e os sujeitos não se beneficiaram da formalização explícita dessas relações durante a interação com o adulto ${ }^{(30)}$.

Com o objetivo de investigar a natureza e a quantidade de informações estocadas na memória após uma única exposição a uma nova palavra e seu referente, Dollaghan ${ }^{(16)}$ realizou algumas adaptações ao paradigma do mapeamento rápido proposto inicialmente por Carey e Bartlett. A autora avaliou o desempenho de 35 crianças em desenvolvimento normal de linguagem na faixa etária de dois a cinco anos em um procedimento experimental que consistia na apresentação de uma não-palavra e seu respectivo referente durante um jogo. A não-palavra era produzida apenas uma vez pelo examinador, e em seguida eram realizadas tarefas de compreensão, produção e reconhecimento da nova palavra, e ainda uma tarefa de localização, que tinha o objetivo de verificar se a criança havia mapeado informações contextuais não-linguísticas sobre o objeto. Os resultados mostraram que, de fato, as crianças em idade pré-escolar parecem realizar o mapeamento rápido de informações linguísticas e não-linguísticas após poucas exposições a novas palavras, uma vez que $81 \%$ dos sujeitos foi capaz de compreender, $45 \%$ foi capaz de produzir pelo menos dois dos três fonemas que compunham a não-palavra, $62 \%$ dos que não nomearam reconheceram a nova palavra e $61 \%$ foi capaz de indicar a localização do referente.

Outros autores ${ }^{(18)}$ também investigaram a habilidade de mapeamento rápido, porém o procedimento experimental diferenciou-se substancialmente do realizado por Dollaghan ${ }^{(16)}$ : os sujeitos eram mais novos (16 a 18 meses), o número de palavras ensinadas foi maior (24 palavras de baixa frequência e desconhecidas pelos sujeitos), assim como o número de apresentações (que variou entre os quatro conjuntos de palavras em função da prática - alta, média ou baixa -, ao longo de 12 sessões) e a quantidade de suporte contextual oferecido (contexto ostensivo e não-ambíguo). Como medida de desempenho, apenas uma tarefa de compreensão foi realizada. A proposta do estudo era examinar os efeitos da prática na acessibilidade das palavras. Os resultados mostraram que a prática com as palavras que foram apresentadas mais vezes levou à aquisição mais rápida das palavras que foram apresentadas menos vezes, sugerindo que o desenvolvimento do vocabulário é um processo contínuo de ajuste do sistema lexical para permitir maior acessibilidade às informações já armazenadas. Esse processo gradual de automatização permitiria que as crianças concentrassem seus esforços em tarefas linguísticas mais complexas, como a aquisição da gramática.

\section{Desempenho de crianças com AEDL em tarefas de ma- peamento rápido}

Utilizando os mesmos parâmetros e paradigmas desenvolvidos em estudos experimentais com enfoque na aquisição lexical de crianças em desenvolvimento normal de linguagem, vários pesquisadores têm procurado determinar as razões da falha dos sujeitos com AEDL no que se refere às mudanças no ritmo de desenvolvimento do vocabulário e aos princípios e mecanismos que operam na fase de maior assimilação de novas palavras, permitindo que sejam elaborados procedimentos de intervenção que facilitem a aquisição de crianças com alterações nesse desenvolvimento.

As maiores evidências de que a aprendizagem de palavras é difícil para os pré-escolares com AEDL vêm de estudos que investigaram a compreensão inicial das novas palavras, comparando o desempenho de crianças com esse diagnóstico e de crianças em desenvolvimento normal de linguagem em tarefas de mapeamento rápido e de exposição mínima às palavras e seus referentes (ambas situações nãoostensivas de apresentação de palavras) ou em tarefas com maior suporte contextual (situações ostensivas, desenvolvidas para a reprodução de contextos comumente utilizados na intervenção) $)^{(26,35-38)}$.

Estudos baseados nas premissas do mapeamento rápido de novos itens lexicais em crianças com AEDL indicam que seu desempenho em tarefas desse tipo é pior do que o de seus pares em desenvolvimento normal, em vários aspectos: essas crianças apresentam piores desempenhos em tarefas de compreensão e produção de novas palavras, e necessitam de maior número de exposições e maior suporte contextual, além de enfoque em um menor número de itens, para adquirilos. Entretanto, o potencial de aprendizagem de palavras em crianças com AEDL pode ser melhor do que o indicado pela sua performance nessas tarefas de mapeamento rápido, dependendo da quantidade de suporte oferecida pelo interlocutor na tarefa proposta ${ }^{(35)}$.

Os resultados de pesquisas em que havia grande suporte contextual durante a exposição mostraram que as crianças com AEDL, como um grupo, foram menos eficientes na produção das novas palavras do que as crianças em desenvolvimento normal. Entretanto, isso não ocorreu para todos os sujeitos, havendo grande variação individual, o que levou à conclusão de que a compreensão não garante a produção dos novos itens lexicais. Além disso, apesar do número de exposições não ter variado entre os sujeitos, as crianças em desenvolvimento normal aprenderam mais palavras do que as crianças com alterações de linguagem ${ }^{(36)}$. 


\section{Processamento da informação e influências na aquisição lexical}

O processo de reforço e complementação das representações fonológicas e semânticas correspondentes a uma nova palavra a cada nova experiência deve ocorrer tanto em crianças em desenvolvimento normal quanto crianças com AEDL, e a qualidade das representações criadas constitui um fator determinante das habilidades linguísticas ${ }^{(28)}$. Habilidades reduzidas em reconhecer as informações relevantes para o mapeamento do significado no contexto em que uma nova palavra é apresentada resultam em representações semânticas empobrecidas. Por outro lado, falhas na codificação das informações fonéticas que representam as palavras resultam em léxico reduzido ${ }^{(37)}$.

Em busca de marcadores clínicos para as AEDL, alguns estudos têm investigado as habilidades de processamento linguístico, que estão diretamente relacionadas à criação das representações lexicais ${ }^{(6)}$. Considerados em conjunto, os resultados desses estudos sugerem que as crianças com AEDL apresentam uma subespecificação de suas representações fonológicas, que são mais gerais e menos refinadas do que as de crianças em desenvolvimento normal de linguagem, em decorrência de uma capacidade limitada de processamento da informação, que se manifesta nas pobres habilidades de memória operacional fonológica e na velocidade reduzida de processamento em tarefas cognitivas em geral ${ }^{(3,28,36)}$.

Outros autores estudaram as características das representações semânticas de crianças com DEL ${ }^{(37-39)}$ e concluíram que existe um paralelo entre as dificuldades encontradas na aprendizagem das representações fonéticas e no mapeamento rápido das características semânticas correspondentes. Um estudo ${ }^{(37)}$ verificou a compreensão de crianças entre quatro e seis anos com DEL no que se refere às características semânticas de objetos e ações desconhecidos, em comparação com sujeitos em desenvolvimento normal de linguagem. Os resultados mostraram que as crianças com DEL tiveram pior desempenho tanto no reconhecimento das características semânticas quanto dos nomes dos objetos, sugerindo que as dificuldades descritas na aquisição lexical desses sujeitos envolvem as duas formas de representação necessárias para o estabelecimento de um novo item lexical.

Esses resultados corroboraram estudos anteriores ${ }^{(39)}$, que usaram tarefas de desenho e definição de palavras reais de baixa frequência (porém adequadas para a faixa etária dos sujeitos), em comparação com o desempenho em uma tarefa de nomeação, para verificar a qualidade das representações semânticas de crianças com DEL. Eles observaram que os desenhos e as definições dos sujeitos eram significantemente mais pobres naqueles itens que eram nomeados incorretamente pelos sujeitos e que as crianças com DEL tiveram, de maneira geral, desempenho significantemente pior do que seus pares de mesma idade cronológica, demonstrando representações semânticas limitadas e correspondências pobres destas com as representações fonológicas, possivelmente relacionadas a pobres habilidades de memória operacional fonológica.

Outro estudo $^{(38)}$ obteve resultados análogos em tarefas da mesma natureza, porém realizadas após um procedimento experimental de ensino de novas palavras. Também essa autora concluiu que a dificuldade na aquisição lexical parece estar relacionada tanto à apreensão dos traços fonológicos quanto semânticos, conclusão baseada nos vários padrões de combinação dos desempenhos dos sujeitos nas diferentes tarefas realizadas, sugerindo ainda que a produção das novas palavras, mais do que sua compreensão, é o maior impedimento para o sucesso da aquisição lexical das crianças com DEL. A partir desses resultados, e utilizando o mesmo paradigma para a análise das habilidades, a mesma autora propôs um novo estudo, cujo objetivo era verificar se pistas semânticas ou fonológicas beneficiam a aquisição lexical de crianças com DEL, em comparação com crianças em desenvolvimento normal de linguagem ${ }^{(40)}$. Os resultados mostraram que de fato o uso de pistas facilita a aquisição lexical de crianças com DEL, porém diferentes tipos de pistas auxiliam diferentes aspectos desse processo: as pistas semânticas beneficiaram a compreensão das novas palavras e as pistas fonológicas melhoraram o desempenho na tarefa de nomeação. Para as crianças normais, ao contrário, os dois tipos de pistas tiveram o mesmo efeito. Os dois grupos se diferenciaram em relação ao número de palavras que compreenderam e produziram em todas as condições (os normais tiveram melhor desempenho do que os DEL), com exceção do desempenho na tarefa de produção após o treino com pistas fonológicas.

Os estudos sobre as representações mentais do léxico comprovam, assim, uma forte relação entre os déficits de armazenamento das informações semânticas e fonológicas, incluindo as dificuldades na organização e categorização dos referentes, e o desempenho em tarefas que exigem o acesso e a produção do material armazenado ${ }^{(12)}$. Ao mesmo tempo, as representações semânticas e fonológicas são refinadas à medida que o vocabulário aumenta; portanto, quanto maior o número de palavras aprendidas, melhor a habilidade de acrescentar novas palavras, tanto pelas relações semânticas e fonológicas comparativas estabelecidas quanto pela prática (treino) das habilidades necessárias para processar as novas informações. Embora a frequência, as similaridades fonológicas com outras palavras e a regularidade morfológica auxiliem o acesso e a produção das novas palavras, as crianças com AEDL utilizam processos diferentes das crianças normais para analisá-las e armazená-las e, de maneira geral, fazem isso de forma mais lenta. Assim, vários fatores interagem entre si e parecem ser determinantes para as dificuldades das crianças com AEDL na aquisição lexical, especificamente, e nos progressos linguísticos subsequentes ${ }^{(35)}$.

\section{DISCUSSÃO}

O levantamento bibliográfico realizado para o presente estudo revelou o caminho que as pesquisas internacionais sobre a aquisição lexical têm seguido. Estudos com objetivos e metodologias diferentes, de natureza observacional ou experimental, e até mesmo com embasamentos teóricos diversos, têm contribuído para o esclarecimento de questionamentos frequentemente observados por linguistas, psicólogos do desenvolvimento e fonoaudiólogos do mundo todo. Os resultados do caminho percorrido por essas pesquisas permitem, atualmente, não só a descrição mais precisa do que deve ser 
o desenvolvimento do vocabulário, mas também o que pode ser considerado desviante.

Os estudos mostram que a aquisição da linguagem, e do vocabulário, em particular, é extremamente complexa, passível de influências e interferências do ambiente no qual a criança está inserida, das relações sociais estabelecidas, e de características particulares de cada indivíduo no que se refere às habilidades cognitivas, de funções executivas e de memória, e à própria relação com a linguagem, em todos os seus aspectos ${ }^{(41)}$. Ainda assim, o panorama do processo normal de aquisição do vocabulário contribui para o estabelecimento de diagnósticos cada vez mais precisos e, principalmente, de recursos terapêuticos mais eficazes.

Os estudos mostram que crianças com AEDL apresentam as mesmas tendências na aquisição lexical e são influenciadas da mesma maneira por fatores lexicais e semânticos que crianças em desenvolvimento normal de linguagem. Dessa forma, essas crianças apresentam maior facilidade na aquisição de palavras de classe aberta (a compreensão e a produção dos nomes de objetos são maiores do que as mesmas habilidades com relação às palavras referentes a ações, embora essa diferença seja menos marcada do que para as crianças em desenvolvimento normal de linguagem), com maior frequência de ocorrência na língua, e que obedeçam às restrições fonológicas e métricas de seleção lexical (no início do desenvolvimento, palavras cujas consoantes iniciais já fazem parte de seu inventário fonológico, com sílabas simples). Além disso, elas também apresentam melhor desempenho em tarefas de compreensão do que em tarefas de produção de novas palavras (compreensão precede a produção), e estão sujeitas aos mesmos princípios de aquisição (embora estes sofram grandes interferências das habilidades de processamento da informação): elas são capazes de mapear o nome desconhecido ao seu referente com base no seu conhecimento anterior da categoria a que o objeto conhecido pertence ${ }^{(29)}$, assumem o princípio da exclusividade mútua (de que a cada objeto corresponde um nome) quando diante de referentes não categorizados ${ }^{(26,31)}$, e são influenciadas pela intenção referencial de seus interlocutores ${ }^{(34)}$.

As diferenças entre crianças em desenvolvimento normal e crianças com AEDL no processo de aquisição lexical são observadas em habilidades e/ou características influenciadas ou diretamente relacionadas aos mecanismos envolvidos no processamento da informação, que comprometem a qualidade e a recuperação das representações fonológicas e semânticas correspondentes a um novo item lexical, e justificam as alterações de vocabulário que constituem uma das marcas clínicas das AEDL: habilidades de categorização hierárquica das palavras, de memória operacional fonológica, de reconhecimento e decisão lexical, e ainda de variações na quantidade e na qualidade do input (velocidade, frequência, intervalo, prosódia, uso de gestos e ênfase). Assim, as crianças com AEDL apresentam representações fonológicas mais gerais e menos refinadas do que as de crianças em desenvolvimento normal de linguagem, em decorrência de uma capacidade limitada de processamento da informação( ${ }^{(3)}$.

Considerando os resultados desses estudos em conjunto, é possível sugerir que as crianças com AEDL são favorecidas, no que refere à facilidade para aquisição lexical, por situações em que são expostas ostensivamente e com grande suporte contextual a um menor número de palavras.

\section{COMENTÁRIOS FINAIS}

A revisão de literatura apresentada neste estudo mostrou grande variedade de achados relacionados ao desenvolvimento do vocabulário, abrangendo as tendências e variações e também outras habilidades envolvidas no processo de aquisição lexical.

De maneira geral, os resultados sugerem que as alterações lexicais que constituem um dos marcos inicialmente observados em crianças com AEDL são justificadas por dificuldades observadas em habilidades influenciadas ou diretamente relacionadas aos mecanismos envolvidos no processamento da informação, que comprometem a qualidade e a recuperação das representações fonológicas e semânticas correspondentes a um novo item lexical.

Entretanto, vários estudos sugerem que situações ostensivas e de grande suporte contextual que enfoquem poucas novas palavras favorecem a aquisição lexical de crianças com AEDL.

\begin{abstract}
The aim of the present study was to describe the similarities and differences found throughout lexical acquisition between normally developing children and children with developmental language disorder through an extensive literature review. The search was carried out in the databases SciELO, Lilacs, PubMed, Web of Science and Dedalus, and covered the last decades of studies in the area. The selected studies, of observational or experimental nature, showed great variability of findings related to vocabulary development, describing tendencies and variations, and also other abilities enrolled in the lexical acquisition process. Generally, the results suggest that the lexical difficulties that constitute one of the first alterations observed in children with developmental language disorder are justified by difficulties in abilities and/or characteristics influenced or directly related to mechanisms involved in information processing, which compromise the quality and the retrieval of phonological and semantic representations corresponding to a new lexical item. However, many studies suggest that ostensive situations and great contextual support emphasizing few novel words facilitate the lexical acquisition of children with developmental language disorder.
\end{abstract}

Keywords: Child language; Language development; Language development disorders; Vocabulary; Review literature as topic 


\section{REFERÊNCIAS}

1. Reed V. Associations between phonology and other language components in children's communicative performance: clinical implications. Aust J Hum Commun Disord. 1992;20(2):75-87.

2. Benedict H. Early lexical development: comprehension and production. J Child Lang. 1979;6(2):183-200.

3. Bishop DV. The underlying nature of specific language impairment. J Child Psychol Psychiatry. 1992;33(1):3-66. Review.

4. Rescorla L, Mirak J, Singh L. Vocabulary growth in late talkers: lexical development from 2;0 to 3;0. J Child Lang. 2000;27(2):293-311.

5. Leonard LB, Ellis Weismer S, Miller CA, Francis DJ, Tomblin JB, Kail $\mathrm{RV}$. Speed of processing, working memory, and language impairment in children. J Speech Lang Hear Res. 2007;50(2):408-28.

6. Conti-Ramsden G. Processing and linguistic markers in young children with specific language impairment (SLI). J Speech Lang Hear Res. 2003;46(5):1029-37.

7. Befi-Lopes DM, Puglisi ML, Rodrigues A, Giusti E, Gândara JP, Araújo K. Perfil comunicativo de crianças com alterações específicas no desenvolvimento da linguagem: caracterização longitudinal das habilidades pragmáticas. Rev Soc Bras Fonoaudiol. 2007;12(4):265-73.

8. Bassano D, Maillochon I, Eme E. Developmental changes and variability in the early lexicon: a study of French children's naturalistic productions. J Child Lang. 1998;25(3):493-531.

9. Bloom P. Précis of How children learn the meanings of words. Behav Brain Sci. 2001;24(6):1095-103; discussion 1104-34.

10. D'Odorico L, Fasolo M. Nouns and verbs in the vocabulary acquisition of Italian children. J Child Lang. 2007;34(4):891-907.

11. Schwartz RG, Leonard LB. Lexical imitation and acquisition in language-impaired children. J Speech Hear Disord. 1985;50(2):141-9.

12. German DJ, Newman RS. The impact of lexical factors on children's word-finding errors. J Speech Lang Hear Res. 2004;47(3):624-36.

13. Schwartz RG, Leonard LB. Do children pick and choose? An examination of phonological selection and avoidance in early lexical acquisition. J Child Lang. 1982;9(2):319-36.

14. Dobrich W, Scarborough HS. Phonological characteristics of words young children try to say. J Child Lang. 1992;19(3):597-616.

15. Goldin-Meadow S, Seligman ME, Gelman R. Language in the two-year old. Cognition. 1976;4(2):189-202.

16. Dollaghan C. Child meets word: "fast mapping" in preschool children. J Speech Hear Res. 1985;28(3):449-54.

17. Capone NC, McGregor KK. The effect of semantic representation on toddlers' word retrieval. J Speech Lang Hear Res. 2005;48(6):1468-80.

18. Gershkoff-Stowe L, Hahn ER. Fast mapping skills in the developing lexicon. J Speech Lang Hear Res. 2007;50(3):682-97.

19. Gershkoff-Stowe L, Thal DJ, Smith LB, Namy LL. Categorization and its developmental relation to early language. Child Dev. 1997;68(5):84359.

20. Nazzi T, Bertoncini J. Before and after the vocabulary spurt: two modes of word acquisition? Dev Sci. 2003;6(2):136-42.

21. Borovsky A, Elman J. Language input and semantic categories: a relation between cognition and early word learning. J Child Lang. 2006;33(4):759-90.

22. Nguyen SP, Murphy GL. An apple is more than just a fruit: crossclassification in children's concepts. Child Dev. 2003;74(6):1783-806.

23. Waxman SR, Braun I. Consistent (but not variable) names as invitations to form object categories: new evidence from 12-month-old infants. Cognition. 2005;95(3):B59-68.
24. Howell SR, Jankowicz D, Becker S. A model of grounded language acquisition: sensorimotor features improve lexical and grammatical learning. J Mem Lang. 2005;53(2):258-76.

25. Garlock V.M, Walley AC, Metsala JL. Age-of-acquisition, word frequency, and neighborhood density effects on spoken word recognition by children and adults. J Mem Lang. 2001;45(3):468-92.

26. Jarvis LH, Merriman WE, Barnett M, Hanba J, Van Haitsma KS. Input that contradicts young children's strategy for mapping novel words affects their phonological and semantic interpretation of other novel words. J Speech Lang Hear Res. 2004;47(2):392-406.

27. Walley AC. The role of vocabulary development in children's spoken word recognition and segmentation ability. Dev Rev. 1993;13(3):286350 .

28. Maillart C, Schelstraete MA, Hupet M. Phonological representations in children with SLI: a study of French. J Speech Lang Hear Res. 2004;47(1):187-98.

29. Mervis CB, Bertrand J. Acquisition of the novel name-nameless category (N3C) principle. Child Dev. 1994;65(6):1646-62.

30. Deák GO, Wagner JH. "Slow mapping" in children's learning of semantic relations. In: Proceedings of the Annual Conference of the Cognitive Science Society. 25th, 2003, Boston. Boston: CogSci, 2003. p. 318-23.

31. Scofield J, Behrend DA. Two-year-olds differentially disambiguate novel words and facts. J Child Lang. 2007;34(4):875-89.

32. Vouloumanos A, Werker JF. Infants' learning of novel words in a stochastic environment. Dev Psychol. 2009;45(6):1611-7.

33. Samuelson LK, Smith LB. They call it like they see it: spontaneous naming and attention to shape. Dev Sci. 2005;8(2):182-98.

34. Gelman SA, Bloom P. Young children are sensitive to how an object was created when deciding what to name it. Cognition. 2000;76(2):91-103.

35. Rice ML, Oetting JB, Marquis J, Bode J, Pae S. Frequency of input effects on word comprehension of children with specific language impairment. J Speech Hear Res. 1994;37(1):106-22.

36. Weismer SE, Hesketh LJ. Lexical learning by children with specific language impairment: effects of linguistic input presented at varying speaking rates. J Speech Lang Hear Res. 1996;39(1):177-90.

37. Alt M, Plante E, Creusere M. Semantic features in fast-mapping: performance of preschoolers with specific language impairment versus preschoolers with normal language. J Speech Lang Hear Res. 2004;47(2):407-20.

38. Gray S. Word learning by preschoolers with specific language impairment: predictors and poor learners. J Speech Lang Hear Res. 2004;47(5):1117-32.

39. McGregor KK, Newman RM, Reilly RM, Capone NC. Semantic representation and naming in children with specific language impairment. J Speech Lang Hear Res. 2002;45(5):998-1014.

40. Gray S. Word-learning by preschoolers with specific language impairment: effect of phonological or semantic cues. J Speech Lang Hear Res. 2005;48(6):1452-67.

41. Gaskell MG, Ellis AW. Word learning and lexical development across the lifespan. Philos Trans R Soc Lond B Biol Sci. 2009;364(1536):360715 . 